\title{
THE GREENSPAN BOUND FOR THE ORDER OF DIFFERENTIAL SYSTEMS
}

\author{
RICHARD M. COHN ${ }^{1}$
}

\begin{abstract}
Let $S$ be a system of ordinary differential polynomials in indeterminates $y_{1}, \ldots, y_{n}$ and of order at most $r_{i}$ in $y_{i}, 1<i<n$. It was shown by J. F. Ritt that if $\mathscr{T}$ is a component of $S$ of differential dimension 0 , then the order of $\mathscr{R}$ is at most $r_{1}+\ldots+r_{n}$. B. Greenspan improved this bound in the case that every component of $S$ has differential dimension 0 . (His work was carried out for difference equations, but is easily transferred to the differential case.) It is shown that the Greenspan bound is valid without this restriction.
\end{abstract}

0. Notation. The following conventions will be used throughout: $\mathbf{N}$ is the set of natural numbers including 0 . Transcendence degree is denoted by t.d. and differential transcendence degree by d.t.d. Superscripts denote differentiation. $\mathcal{K}\left\{y_{1}, \ldots, y_{n}\right\}$ is a differential polynomial ring over the ordinary differential field $\mathcal{K}$ of characteristic 0 . Where convenient a vector notation will be used, for example, $y$ for $\left(y_{1}, \ldots, y_{n}\right)$. If $\Re$ is a differential manifold over $\mathcal{K}$, then $\Delta \mathscr{T}$ denotes the differential dimension of $\mathscr{N}$; and ord $\mathfrak{N}$ denotes the order of $\mathscr{N}$, that is, t.d. $\mathscr{K}\langle a\rangle / \mathscr{K}$, where $a$ is a generic point of $\mathscr{N}$. The notation for differential kernels follows [4].

1. Introduction. Let $S$ be a set of differential polynomials of $\mathscr{K}\left\{y_{1}, \ldots, y_{n}\right\}$ which is of bounded order in each $y_{i}$. [That is, there exists $r \in \mathbf{N}$ such that no polynomial of $S$ is of order greater than $r$ in $y_{i}, 1<i<n$.] This note is concerned with determining a bound for the order of $S$, that is, an element $B \in \mathbf{N} \cup\{-\infty\}$, dependent only on the orders to which the $y_{i}$ occur in the polynomials of $S$, such that if $\boldsymbol{X}$ is a component of $S$ of dimension 0 , then ord $\boldsymbol{x}<\boldsymbol{B}$. (If $B=-\infty$, this is to mean that $S$ has no component of dimension 0 .)

One such bound was found by Ritt [6, p. 301], [3, p. 194]. Let $r_{i}$ be the maximum of the orders of the polynomials of $S$ in $y_{i}, 1<i<n$. Let $R(S)=\sum_{i=1}^{n} r_{i}$. Ritt showed that one may choose $B=R(S)$. Greenspan $[2,87]$ suggested an improvement for difference polynomials which is easily transferred to the differential case. Let $P \in S, P \neq 0$. Then there exists $h(P) \in \mathrm{N}$ such that $P^{(h(P))}$ is of order at most $r_{i}$ in each $y_{i}$, but $P^{(h(P)+1)}$ is not. (If $P=0$, let $h(p)=0$.) Let $G(S)=R(S)-$ $\max _{S} h(P)$. Following Greenspan one can show that for systems $S$ such that every

Received by the editors July 26, 1978 and, in revised form, September 30, 1979.

AMS (MOS) subject classifications (1970). Primary 12M05.

Key words and phrases. Order of differential systems, differential kernel, Greenspan bound, Jacobi bound.

'The author is very grateful to the referee for numerous suggestions which have improved the presentation of this paper. 
component has dimension 0 , one may choose $B=G(S)$. Using the work of Lando [4] it will now be shown that one may choose $B=G(S)$ without this restriction.

\section{The main theorem.}

TheOREM. Let $S \subseteq \mathcal{K}\left\{y_{1}, \ldots, y_{n}\right\}$ be of bounded order in each $y_{i}$. Let $\mathfrak{T}$ be a component of the manifold of $S$ such that $\Delta \mathfrak{R}=0$. Then ord $\mathfrak{T}<G(S)$.

Proof. Replace $S$ by the equivalent first order system $S^{1}$, and let $\mathfrak{T}^{1}$ be the component of $S^{1}$ which corresponds to $\Re$. Let $u=\left(u_{1}, \ldots, u_{p}\right)$ be a generic point of $\Re^{1} . u$ is a regular realization of the kernel $\Re$ consisting of $\mathcal{K}\left(u, u^{\prime}\right)$ and the obvious derivation. Since $S^{1}$ is a first order system, every realization of $R$ annuls $S^{1}$, and it follows from [4, Corollary to Theorem I] and the fact that $u$ is a generic point that $u$ is a principal realization of $\Re$. Therefore, by [4, Proposition 3] d.t.d. $\mathscr{K}\langle u\rangle / \mathcal{K}=$ t.d. $\mathscr{K}\left(u, u^{\prime}\right) / \mathcal{K}(u)=0$.

REMARK. If all $r_{i}=0$, let $S^{I}=S$, and let $\Re$ be the kernel of length 0 consisting of $\mathscr{K}(u)$ and the derivation of $\mathcal{K}$. It is easy to adapt the following reasoning to this case.

Let $u_{1}, \ldots, u_{k}$ be those coordinates of $u$ which correspond to indeterminates occurring only to order 0 in $S$. Then $u_{1}, \ldots, u_{k}$ correspond to indeterminates occurring only to order 0 in $S^{1}$. Evidently $R(S)=p-k$.

Let $w=\left(u_{k+1}, \ldots, u_{p}\right)$. It will be shown that $u_{1}, \ldots, u_{k}$ are algebraic over $\mathcal{K}(w)$. Suppose this is not so. Let $d$ denote the contraction of the derivation of $\Re$ to a derivation from $\mathscr{K}(w)$ into $\mathscr{K}\left(w, w^{\prime}\right)$. By standard theorems on extending derivations $d$ extends to a derivation $d^{*}$ carrying $\left(u_{1}, \ldots, u_{k}\right)$ to $v=\left(v_{1}, \ldots, v_{k}\right)$ with t.d. $\mathscr{K}\left(u, v, w^{\prime}\right) / \mathscr{K}(u)>0$, and with $\left(u, v, w^{\prime}\right)$ specializing to $\left(u, u^{\prime}\right)$. Let $u^{*}$ denote $\left(v, w^{\prime}\right)$. Then $\Re^{*}=\mathscr{K}\left(u, u^{*}\right)$ with derivation $d^{*}$ is a kernel. Let $z$ be a principal realization of $\Re^{*}$. Because no proper derivatives of the indeterminates corresponding to $u_{1}, \ldots, u_{k}$ appear in the differential polynomials in $S^{1}, z$ is a solution of $S^{1}$. By [4, Theorem 1] there is a differential specialization of $z$ to $u$ over $\mathcal{K}$. This specialization is not generic since $u$ is by hypothesis differentially algebraic over $\mathcal{K}$ but $z$ is not [4, Proposition 3]. This contradicts the choice of $u$ as a generic point of a component of $S^{1}$. The claim made at the start of this paragraph is established. Since d.t.d. $\mathscr{K}\langle u\rangle / \mathcal{K}=0$, it follows using [4, Proposition 3] that ord $\mathscr{K}^{I}=$ t.d. $\mathscr{K}\langle u\rangle / \mathcal{K}=$ t.d. $\mathscr{K}\left(u, u^{\prime}\right) / \mathscr{K}=$ t.d. $\mathscr{K}\left(u, u^{\prime}\right) / \mathscr{K}(u)+$ t.d. $\mathscr{K}(u) / \mathcal{K}=$ t.d. $\mathscr{K}(u) / \mathcal{K}=$ t.d. $\mathscr{K}(w) / \mathscr{K}<R(S)$.

Let $P \in S$. It will be shown that t.d. $\mathscr{K}(w) / \mathscr{K}<R(S)-h(P)$. This will complete the proof of the theorem. From the result of the preceding paragraph, we may assume $h(P)>0$; and so $P \neq 0$. We choose a ranking of the indeterminates. Let $P_{0}=P$, and let $P_{i}$ be the separant of $P_{i-1}, i=1,2, \ldots$ Choose $Q=P_{j}$, where $j$ is such that $P_{0}, \ldots, P_{j} \in I(\mathscr{T}), P_{j+1} \notin I(\Re)$. Let $F_{i}$ be obtained from $Q^{(i)}$ by the substitution which produces $S^{I}$ from $S$. Since $h(P)>0$, the $F_{i}$ do not involve $u_{1}, \ldots, u_{k}$. From the construction of the $F_{i}$ it is easy to see that each $F_{i}$, $0<i<h(P)-1$, involves an indeterminate $t_{i}$ not present in any $F_{j}, j<i$, and such that $\partial F_{i} / \partial t_{i} \notin I\left(\mathscr{N}^{I}\right)$. Since $w$ annuls the $F_{i}, 0<i<h(P)-1$, it follows that the coordinates of $w$ corresponding to the $t_{i}$ are algebraically dependent over $\mathscr{K}$ on 
the remaining coordinates. Hence, t.d. $\mathscr{K}(w) / \mathcal{K}<R(S)-h(P)$ as claimed.

3. The case $n=$ card $S$. Let $S=\left\{S_{1}, \ldots, S_{n}\right\}$ and let $r_{i j}^{*}$ be the order of $S_{i}$ in $y_{j}$. $\left(r_{i j}^{*}=0\right.$ if $y_{j}$ does not appear in $S_{i}$.) Let $J^{*}(S)=\max _{j} \sum_{i=1}^{n} r_{i, j(i)}^{*}$, where $j$ runs through all permutations of $(1, \ldots, n)$. Let $r_{i j}=r_{i j}^{*}$ if $y_{j}$ appears effectively in $S_{i}, r_{i j}=-\infty$ otherwise; and let $J(S)=\max _{j} \sum_{i=1}^{n} r_{i, j(i)}$, where $j$ runs through all permutations. Ritt [6] called $J(S)$ the Jacobi bound after its proposer, and conjectured that it is a bound for the order, a conjecture which has not been established, and whose proof or disproof appears difficult. Lando [4, p. 133] distinguished $J^{*}$ and $J$ as the (weak) Jacobi bound and the strong Jacobi bound respectively. (In her notation, $J$ is the weak bound, $J^{\prime}$ the strong.) We shall be concerned hereafter with the smallest possible bounds obtainable from either the matrix $\left(r_{i j}^{*}\right)$ or the matrix $\left(r_{i j}\right)$. Lando $[4,86]$ showed that no bound smaller than $J^{*}$ or $J$ can be obtained from $\left(r_{i j}^{*}\right)$ or $\left(r_{i j}\right)$ respectively.

In the discussion of the next two paragraphs it is also assumed in obtaining a bound from $\left(r_{i j}^{*}\right)$ that one is given the additional information that no $S_{i}$ is 0 . Easy modifications cover the more general case in which it is known which $S_{i}$ are 0 . These reservations are needed because $h(P)=0$ if $P=0$ so that one cannot determine a bound for $G(S)$ as in the next paragraph.

Given $\left(r_{i j}\right)$ for $S$ we may compute $G(S)$. Given only $\left(r_{i j}^{*}\right)$ we cannot determine $G(S)$, since we do not know which indeterminates are missing from which polynomials. Proceeding as if all indeterminates are present in all the polynomials of $S$ (equivalently as if $\left(r_{i j}^{*}\right)=\left(r_{i j}\right)$ ) we compute an estimate $G^{*}(S)$. Then $G(S)<$ $G^{*}(S)$. Evidently $G$ and $G^{*}$ are bounds obtainable from $\left(r_{i j}\right)$ and $\left(r_{i j}^{*}\right)$ respectively.

Lando [4, Theorem 3] has shown that $J^{*}(S)$ is a bound if all $r_{i j}^{*}<1$. For arbitrary $S$ with $n=$ card $S$, let $S^{1}$ be the corresponding first order system. Then $n^{1}=$ card $S^{1}$, where $n^{1}$ is the number of indeterminates in $S^{1}$, and $J^{*}\left(S^{1}\right)$ is defined, and is a bound for $S$. We denote it by $L^{*}(S)$. Hence, two bounds are at present known which depend on $\left(r_{i j}^{*}\right)$, namely $G^{*}(S)$ and $L^{*}(S)$. A basic tool needed to establish both these bounds (but not the bound $R(S)$ ) is Lando's Specialization Theorem [4, Theorem 1]. For comparison, consider the systems $S^{\prime}, S^{\prime \prime}$ with $\left(r_{i j}^{*}\right)$ matrices

$$
A^{\prime}=\left|\begin{array}{lll}
2 & 2 & 2 \\
2 & 2 & 2 \\
0 & 0 & 0
\end{array}\right|, \quad A^{\prime \prime}=\left|\begin{array}{lll}
2 & 2 & 2 \\
2 & 0 & 0 \\
2 & 0 & 0
\end{array}\right|
$$

respectively. Then $J^{*}\left(S^{\prime}\right)=4, G^{*}\left(S^{\prime}\right)=4, L^{*}\left(S^{\prime}\right)=5 ; J^{*}\left(S^{\prime \prime}\right)=4, L^{*}\left(S^{\prime \prime}\right)=$ $5, G^{*}\left(S^{\prime \prime}\right)=6$.

There is an equivalence between $J$ and $J^{*}$. Let $T$ denote a set of differential polynomials in $\mathscr{K}\left\{y_{1}, \ldots, y_{n}\right\}$. We define $s_{i}(T), 1<i<n$, to be the set of differential polynomials obtained from those of $T$ by replacing $y_{i}^{(n)}$ by $y_{i}^{(j+1)}, j=$ $0,1, \ldots$ Let $F=\left\{T_{i}, i \in I\right\}$, each $T_{i}$ a set of $n$ differential polynomials in $\mathscr{K}\left\{y_{i}, \ldots, y_{n}\right\}$. If $F$ is closed under the $s_{i}, 1<i<n$, then it is easy to show that $J$ provides a bound for the orders of the $T_{i}$ if $J^{*}$ does.

We note, finally, two special cases. Ritt [7] proved that if either all polynomials 
of $S$ are linear or card $S<2$, then $J(S)$ is a bound (and therefore, by Lando's result above, the smallest bound obtainable from $\left.\left(r_{i j}\right)\right)$. When card $S<2, G(S)=$ $J(S)$, provided $J(S)$ is finite, so that this result of Ritt's is generalized in part by the theorem of this paper.

4. Related problems. For older theorems on the (effective) order of systems of difference equations see [1, pp. 252-259], [2], and [5]. Most of the above can readily be transferred and needs no special comment. In particular, the theorem of $\$ 2$ can at once be carried over to difference algebra with the effective order [1, p. 134] of $\mathfrak{T}$ replacing order and initials replacing separants. The characteristic need not be 0 .

For results and conjectures concerning systems of partial differential equations see [3, pp. 194-202] and [8].

\section{REFERENCES}

1. R. M. Cohn, Difference algebra, Interscience, New York, 1965.

2. B. Greenspan, $A$ bound for the orders of the components of a system of algebraic difference equations, Pacific J. Math. 9 (1959), 473-486.

3. E. R. Kolchin, Differential algebra and algebraic groups, Academic Press, New York and London, 1973.

4. B. A. Lando, Jacobi's bound for the order of systems of first order differential equations, Trans. Amer. Math. Soc. 152 (1970), 119-135.

5. __ Jacobi's bound for first order difference equations, Proc. Amer. Math. Soc. 32 (1972), 8-12.

6. J. F. Ritt, Systems of algebraic differential equations, Ann. of Math. (2) 36 (1935), 293-302.

7. _ Jacobi's problem on the order of a system of differential equations, Ann. of Math. (2) 36 (1935), 303-312.

8. J. Tomasovic, A generalized Jacobi conjecture for partial differential equations, Trans. Amer. Math. Soc. (to appear).

Departiment of Mathematics, Rutgers University, New Brunswick, New Jersey 08903 